\title{
Endoscopic full-thickness resection of gastric gastrointestinal stromal tumor: a Japanese case series
}

\author{
Satoki Shichijo a, Noriya Uedo a, Yoshitomo Yanagimoto ${ }^{b}$, Kazuyoshi Yamamoto ${ }^{b}$, Mitsuhiro Kono ${ }^{a}$, \\ Hiromu Fukuda ${ }^{a}$, Yusaku Shimamoto ${ }^{a}$, Kentaro Nakagawa ${ }^{a}$, Masayasu Ohmoria, Masamichi Arao ${ }^{a}$, \\ Taro Iwatsubo ${ }^{a}$, Hiroyoshi Iwagamia , Shuntaro Inoue ${ }^{a}$, Kenshi Matsuno ${ }^{a}$, Noriko Matsuuraa, \\ Hiroko Nakahiraa , Akira Maekawaa , Takashi Kanesaka ${ }^{a}$, Yoji Takeuchia, Koji Higashino ${ }^{a}$, \\ Takeshi Ohmori', Ryu Ishihara ${ }^{a}$ \\ Osaka International Cancer Institute, Japan
}

\section{Abstract}

\begin{abstract}
Background Gastrointestinal stromal tumors (GISTs) are potentially malignant and are indicated for resection. The standard treatment for resectable GISTs is surgery, although endoscopic resection has been reported outside Japan. This study retrospectively analyzed the results of endoscopic resection of GISTs in Japan.
\end{abstract}

Method We identified patients with GISTs treated only by endoscopic resection in our institute between January 2016 and December 2018, and analyzed their clinical and pathological characteristics.

Results During the study period, 8 GISTs were resected only by endoscopy: 7 were located in the upper third of the stomach and 1 in the middle. All were intraluminal growth type. Median (range) tumor diameter was $20(10-35) \mathrm{mm}$. All tumors were resected en bloc with a median (range) operation time of 67.5 (50-166) min. Complete perforation occurred in 5 cases, but the serosa remained in 2 and the outer layer of the muscularis propria remained in 1 . The defect was endoscopically closed with clip-and-endoloop purse-string suturing $(n=3)$, simple endoclipping $(n=2)$, or over-the-scope clipping $(n=2)$, and 1 did not require closure because the outer longitudinal muscle was preserved. Oral feeding was commenced on postoperative day (POD) 3 (median; range 2-4), and the patient was discharged on POD 6 (median; range 4-11). No serious adverse event developed after the procedures.

Conclusion Endoscopic resection for selected cases of small intraluminal GISTs is feasible, making it a viable alternative treatment option to laparoscopic surgery.

Keywords Endoscopic resection, endoscopic submucosal dissection, gastrointestinal stromal tumor, endoscopic full-thickness resection

Ann Gastroenterol 2019; 32 (6): 1-7
${ }^{\mathrm{a} D e p a r t m e n t ~ o f ~ G a s t r o i n t e s t i n a l ~ O n c o l o g y ~(S a t o k i ~ S h i c h i j o, ~ N o r i y a ~}$ Uedo, Mitsuhiro Kono, Hiromu Fukuda, Yusaku Shimamoto, Kentaro Nakagawa, Masayasu Ohmori, Masamichi Arao, Taro Iwatsubo, Hiroyoshi Iwagami, Shuntaro Inoue, Kenshi Matsuno, Noriko Matsuura, Hiroko Nakahira, Akira Maekawa, Takashi Kanesaka, Yoji Takeuchi, Koji Higashino, Ryu Ishihara); ${ }^{b}$ Department of Gastroenterological Surgery (Yoshitomo Yanagimoto, Kazuyoshi Yamamoto, Takeshi Ohmori $\left.{ }^{\mathrm{b}}\right)$, Osaka International Cancer Institute, Japan

\section{Conflict of Interest: None}

Correspondence to: Noriya Uedo, Department of Gastrointestinal Oncology, Osaka International Cancer Institute, 3-1-69 Otemae, Chuo-ku, Osaka 541-8567, Japan, e-mail: noriya.uedo@gmail.com

Received 10 May 2019; accepted 13 July 2019; published online 23 September 2019

DOI: https://doi.org/10.20524/aog.2019.0413

\section{Introduction}

Gastric gastrointestinal stromal tumors (GISTs) are potentially malignant and are indicated for resection. According to the clinical practice guideline for GISTs in Japan, $3^{\text {rd }}$ edition 2014, issued by the Japan Society of Clinical Oncology [1], gastric submucosal tumors (SMTs) that: 1) are histologically proven as GIST; 2) are $>5 \mathrm{~cm}$ in size; 3) are of increasing size; or 4) have suspicious malignant findings on gastroscopy, endoscopic ultrasonography or computed tomography, are indicated for resection.

The standard treatment for resectable GISTs is surgery [1-3]. In addition to conventional laparotomy surgery, laparoscopic surgery has been widely performed recently. The oncologic outcome of laparoscopic surgery is reportedly comparable with that of the open approach, and its surgical outcome is superior 
to that of open surgery in terms of low blood loss and short hospital stay [4-7]. Nowadays, laparoscopic and endoscopic cooperative surgery (LECS) can be employed to reduce a gastric wall defect by precise resection with peroral flexible endoscopy using endoscopic submucosal dissection (ESD). In the LECS procedure, the wall defect is closed with laparoscopic suturing. However, as various devices and methods to close the wall defect have been developed, such as the over-the-scope clip (OTSC) $[15,16]$, purse-string suturing $[17,18]$, and lineassisted complete closure (LACC) methods [19-21], reports of the feasibility of endoscopic full-thickness resection (EFTR) for low-grade GISTs have come mainly from China [8-17]. Using this method, the invasiveness of the resection can be minimized without any abdominal wounds. Moreover, EFTR may avoid gastric dysmotility for lesions at the lesser curvature of the stomach, because it does not damage the vagus nerve or vessels in the lesser omentum and avoids excessive partial gastric resection of lesions at the cardia or the pylorus, where local resection by laparoscopic surgery is difficult [18]. Despite these advantages, EFTR for GIST has not been implemented in clinical practice in Japan.

In this study, we retrospectively examined the clinical and pathological characteristics of patients with GISTs treated only by endoscopic resection, and we elucidate the feasibility of EFTR in Japanese practice.

\section{Patients and methods}

\section{Patients and setting}

Patients who underwent resection treatment for gastric GISTs in Osaka International Cancer Institute between January 2016 and December 2018 were retrieved from our pathology database. Among them, patients treated solely by endoscopic resection were enrolled in this study.

The study was approved by the institutional review board. Written informed consent to the procedures was obtained from all patients after explanation of the possible risk of complications, anticipated results, and alternative options such as surgery and no treatment. Informed consent for study participation was waived because of the retrospective nature of the study, and because only anonymous clinical data were used for the analysis.

\section{Indications for resection and pretreatment examination}

Endoscopic ultrasonography and computed tomography scans were performed in all patients. The indications for resection were in accordance with the clinical practice guidelines for GIST in Japan [1]. The resection method was decided on the basis of the growth type, size, and location of the tumor. Essentially, intraluminal growth type tumors less than $4 \mathrm{~cm}$ in size, especially when located near the cardia, were candidates for EFTR or LECS.

\section{Endoscopic procedure}

The procedures were performed in an endoscopy room under deep sedation with intravenous anesthesia in the earlier period of the study; in the later period, the surgery was carried out in an operating room under general anesthesia. All procedures in this study were performed using a gastroscope (EVIS GIFQ260J or GIF-2TQ260M; Olympus, Tokyo, Japan) with a distal attachment (D-201-11804, or D-201-1340; Olympus). A FlushKnife-BT (2.0 mm, DK2618JB; Fujifilm Medical, Tokyo, Japan) [19] and IT knife 2 (KD-611L; Olympus) were used for mucosal incision, submucosal dissection and tumor resection. A Coagrasper (FD-410LR; Olympus) or hot biopsy forceps (Radial jaw 4; Boston Scientific Japan, Tokyo, Japan) was used for hemostasis. A high-frequency electrical generator (VIO 300D; ERBE Elektromedizin, Tübingen, Germany) was used with the following settings: Endocut mode: Effect 2, Duration 3, Interval 3; Swift coagulation mode: Effect 3, $80 \mathrm{~W}$; Soft coagulation mode: Effect 5, $80 \mathrm{~W}$. $\mathrm{CO}_{2}$ insufflation was used in all procedures.

Prior to the ESD, a solution (Mucoup, 0.4\% hyaluronic acid) was injected into the submucosa, the mucosa around the lesion was incised, and the submucosa was dissected until the surface of the tumor was seen (Fig. 1). In some cases, a clip with line (3-0 polyester surgical thread; Natsume Seisakusho, Tokyo, Japan) was used to achieve good visibility for dissecting the submucosal plane and to prevent the tumor being lost from the gastric wall. Submucosal dissection proceeded along the edge of the tumor to discover muscle attachment, after which the muscularis propria was cut along the tumor margin. The resection wound was closed with endoclips (EZ Clip, HX-610-

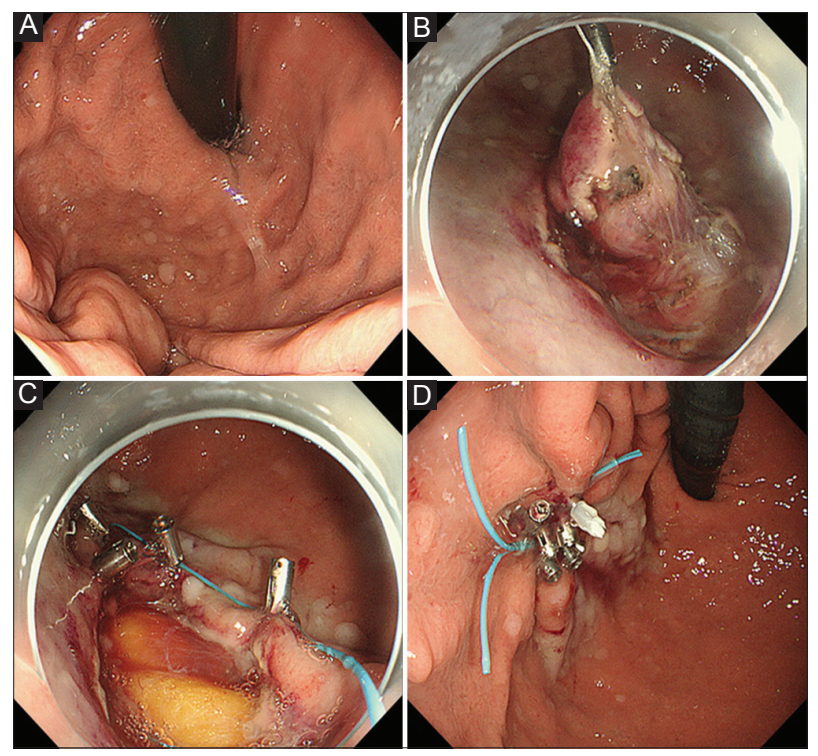

Figure 1 Endoscopic resection of gastric gastrointestinal stromal tumor. (A) A submucosal tumor was located at the greater curvature of the upper body. (B) After submucosal injection of hyaluronic acid, mucosal incision and slight submucosal dissection, a clip with line was attached to facilitate prompt dissection and prevent the tumor from extending beyond the stomach. (C) After complete resection, the perforation site was closed by purse-string suture. (D) The defect was closed completely 
090; Olympus), an OTSC (OTSC System, 13B1X00089050602; Ovesco Endoscopy, Tübingen, Germany) [20,21], a pursestring suture method using an endoloop (HX-400U-30; Olympus) and endoclips [22,23], or the LACC method using nylon string and endoclips [24-26]. Complete closure of the wound was confirmed by careful endoscopic inspection.

\section{Definition of variables}

Patients and tumor information was retrieved from medical records, endoscopic imaging reports, and the pathological database. Descriptions of tumor characteristics were based on the Japanese classification of gastric carcinoma [27]. The procedure time was measured from the start of the submucosal injection to the removal of the lesion. Adverse events were graded according to the Common Terminology Criteria for Adverse Events [28].

\section{Results}

During the study period, a total of 56 patients underwent resection treatment for GIST and 8 patients were treated by endoscopy only (Table 1). The indication for resection was determined by histological diagnosis of GIST in forceps biopsy specimens $(n=3)$ and endoscopic ultrasound-guided fineneedle aspiration $(n=1)$, a tendency to grow in size $(n=1)$, and a malignant finding of heterogeneous parenchyma on endoscopic ultrasonography $(n=3)$. No patients had clinical symptoms.

Five tumors were intended to be removed by flexible endoscopy in an endoscopy room, while 3 were scheduled for LECS in the operating room, but the procedure was completed without laparoscopic closure of the wound. Six lesions were located in the upper third of the stomach, whereas 3 were at the lesser curvature or posterior wall, where the laparoscopic approach was somewhat difficult. Median (range) tumor diameter was 20 (1035) $\mathrm{mm}$. All tumors were resected en bloc, and the median (range) operation time was 67.5 (50-166) min. Complete perforation occurred in 5 cases, but the serosa remained in 2 and the outer layer of the muscularis propria remained in 1. The defect was closed completely using one or a combination of simple clipping $(n=3)$, OTSC $(n=2)$, LACC $(n=1)$, and purse-string suture $(n=2)$. Abdominal paracentesis was not required in any patient.

Antibiotics were administered in 5 patients for several days (mean 3, range 1-7 days), while the remaining 3 patients did not receive antibiotics. No patients complained of spontaneous abdominal pain suggesting peritonitis, except for mild tenderness in a few cases. Oral feeding was commenced on postoperative day (POD) 3 (median; range 2-4). Computed tomography scan or X-ray using oral contrast agent was not routinely performed to confirm complete sealing of the wound. The patient was discharged on POD 6 (median; range 4-11). One patient developed high fever $\left(38.0^{\circ} \mathrm{C}\right)$; otherwise, no $\geq$ Grade 2 adverse event was observed after the procedure.

En bloc resection was achieved in all patients, although 5 of them were regarded as RX resection (i.e., histologically clear margin was indeterminate) on histological examination.

\section{Discussion}

In this retrospective observational study, we investigated small $(\leq 4 \mathrm{~cm})$ intraluminal growth type GISTs treated only by peroral flexible endoscopy. Although many studies of endoscopic treatment for SMTs in other countries have been published [8-17], little has been reported from Japan.

Currently, the standard resection method for gastric GISTs is surgery with negative surgical margins [29]. However, unlike gastric cancer, GISTs, especially when small, require neither lymphadenectomy nor a generous resection margin, because they seldom develop lymph node metastasis and infiltrative growth patterns [30]. In this study, although 5 of 8 patients were histologically diagnosed as RX, this was because of burning of the submucosal tissue near the capsule. All of the tumors were endoscopically removed completely en bloc. In the surgical resection of GIST, the risk of recurrence appears to be largely due to the presence of tumor rupture or intraperitoneal bleeding, but 3 -year recurrence-free survival rates of cases with R0 resection (80\%) and R1 resection without rupture (79\%) are reported to be similar (hazard ratio 0.84 [95\%CI 0.47-1.52], $\mathrm{P}=0.57$ ) [31]. Therefore, in oncologic terms precise local endoscopic resection should be an acceptable treatment for GISTs.

En bloc resection was achieved in all 8 patients with GIST. Principally, the ESD technique was applicable to EFTR. However, we found some procedural differences in EFTR for GISTs from ESD for early gastric cancer (EGC) (Fig. 2). In ESD for EGC, we usually make an incision line approximately $5 \mathrm{~mm}$ outside the boundary of a tumor, whereas in the EFTR procedure the mucosal incision had to be created on the edge or at the center of a tumor. When the mucosa is incised, the mucosal defect becomes larger than actual size of the incision line because of shrinkage of the mucosa, making defect closure very difficult. Therefore, it is better to make the mucosal incision as small as possible in EFTR of GIST. Besides, in ESD for EGC the deep submucosa is dissected along the surface of the muscularis propria, whereas in EFTR of GIST the submucosal dissection is performed along the surface of the tumor. One of the important aims of this deep submucosal dissection in ESD for EGC is to reduce the risk of perforation. When we recognize the surface of the muscularis propria, we can maintain a certain distance between the dissection plane and the muscularis propria, avoiding injury to the muscle surface. Using the same principle, when we recognize the surface of the tumor in EFTR for GIST, we can avoid capsule injury during the submucosal dissection.

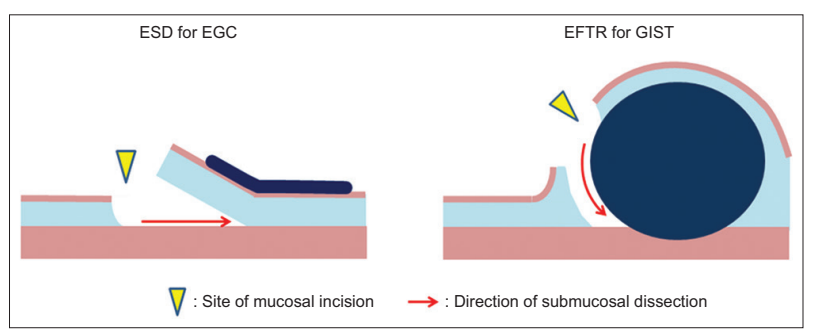

Figure 2 Schema of ESD and EFTR

$E S D$, endoscopic submucosal dissection; EFTR, endoscopic full-thickness resection; GIST, gastrointestinal stromal tumor 


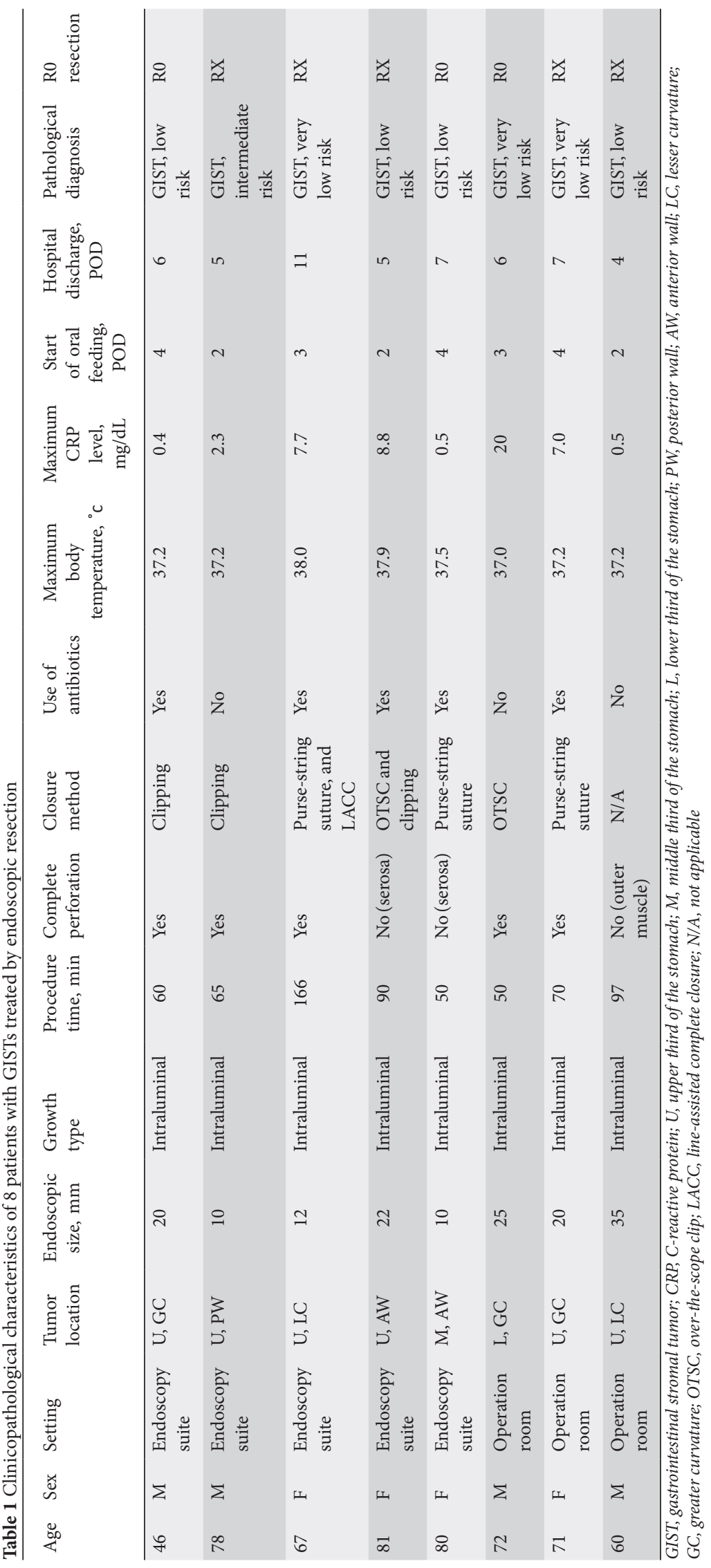


Moreover, we found that this procedure sometimes enabled us to avoid complete perforation. The serosal membrane remained in 2 cases and the outer longitudinal muscle was preserved in 1 patient, making the defect amenable to closure. The clip-andthread method was useful in EFTR for GIST as well as ESD for EGC [32]. With this technique, attachment of the tumor to the muscularis propria was well identified. Especially after creating a full-thickness incision, despite collapse of the gastric lumen, clip-and-thread traction facilitated the identification and cutting of the remnant attachment as well as easy completion of EFTR. Moreover, the clip with line successfully prevented the tumor being lost into the peritoneal cavity, and facilitated tumor retrieval from the stomach. Regarding incomplete perforation and early closure of the muscle defect, pneumoperitoneum was usually mild and abdominal paracentesis was not required in any patient in this case series.

Submucosal tunneling endoscopic resection (STER) is reportedly suitable for resection of esophageal SMTs or SMTs in the cardia [13], but is often unsuitable for gastric GISTs. Because the stomach is not a straight hollow, it is sometimes difficult to create a straight tunnel toward the tumor and orientation may be lost. Moreover, submucosal dissection of the distal pole of the SMT is difficult during a STER procedure, so snare resection is often performed at this juncture. Esophageal leiomyoma, which accounts for almost all esophageal SMTs, is a hard tumor; hence, such snare resection is acceptable, but a gastric GIST is elastic soft and snare resection can be piecemeal. Accordingly, we now regard direct EFTR as a more suitable method for the endoscopic resection of gastric GISTs.

Resection of the tumor is relatively easy for endoscopists familiar with the ESD procedure, but closing the muscle defect is a bottleneck of the EFTR procedure. However, various closure devices and methods have become available recently. For a small or incomplete muscle defect, conventional clipping should suffice. Purse-string suturing [22,23] using endoloop and clips (Fig. 3), or OTSC [20,21] were applied for closure of a larger defect. The purse-string suture requires a 2-channel endoscope, but is an effective and inexpensive method. The OTSC is an easy-to-use and reliable device for closing a defect, but is expensive (about 600 US dollars in Japan) and sometimes requires multiple bites for large defects. Accordingly, we use the purse-string suture as a first-line approach and keep the OTSC as a backup method. Originally developed in our hospital is the LACC method [24-26], whereby a clip with a nylon fishing line is applied through the working channel of a videoendoscope to the mucosa on the proximal side of the defect (Fig. 4A), after which a second clip without line is applied to the mucosa on the distal side of the defect, anchoring the line of the first clip (Fig. 4B,C). The clips are then gathered by pulling on the line (Fig. 4D), and additional clips are applied to make the closure secure and tight (Fig. 4E) [20,21,26]. We usually use this method for closure of a mucosal defect after colorectal ESD, and we used it thus in one case reported here. However, the procedure is somewhat complicated and insecure for closure of a full-thickness gastric wall defect and may be used as an adjunct to repair a remnant hole after purse-string suture or OTSC closure. An endoscopic suturing system (OverStitch; Apollo Endosurgery, Austin, TX, USA) provides secure closure of the gastric wall defect [33], but has not yet been approved by the Japanese Pharmaceutical Affairs regulator, while new suturing devices such as the double-armed bar suturing system (Zeon Medical, Tokyo, Japan) [34] should become available in the future. We believe that standardization of the closure method for muscle defects with a cost-effective procedure should be well addressed before commencing a prospective study to investigate the feasibility of EFTR.

Management of bleeding from serosal vessels is a major difficulty in EFTR procedures [35]. In this case series, however, we did not encounter severe bleeding, which cannot be managed with hemostatic forceps. This was probably because the tumors in our cases were small (median diameter $20 \mathrm{~mm}$ ) and of intraluminal growth type. Extraluminal tumors sometimes have thick feeding arteries from the peritoneum, but when we indicate EFTR for intraluminal gastric GISTs of limited size, the risk of unmanageable bleeding may be considered low.

In the early period of the study, we performed the procedure in endoscopy rooms with the patient under deep sedation. However, patients became restless, especially after full-thickness incision, which made the subsequent procedure unstable and stressful for endoscopists. For this reason, in the later period we

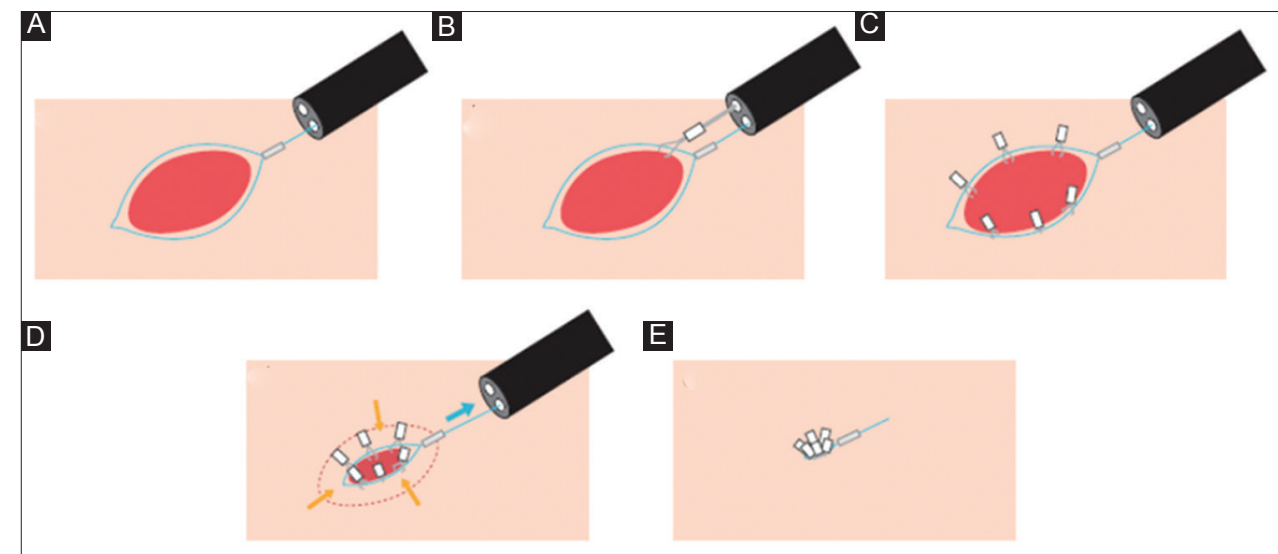

Figure 3 Purse-string suturing using endoloop and clips. (A) An endoloop is placed around the defect. (B) The endoloop is anchored on the normal mucosa with a clip. (C) Clips are applied circumferentially. (D) The endoloop is tied. (E) The defect is closed completely 

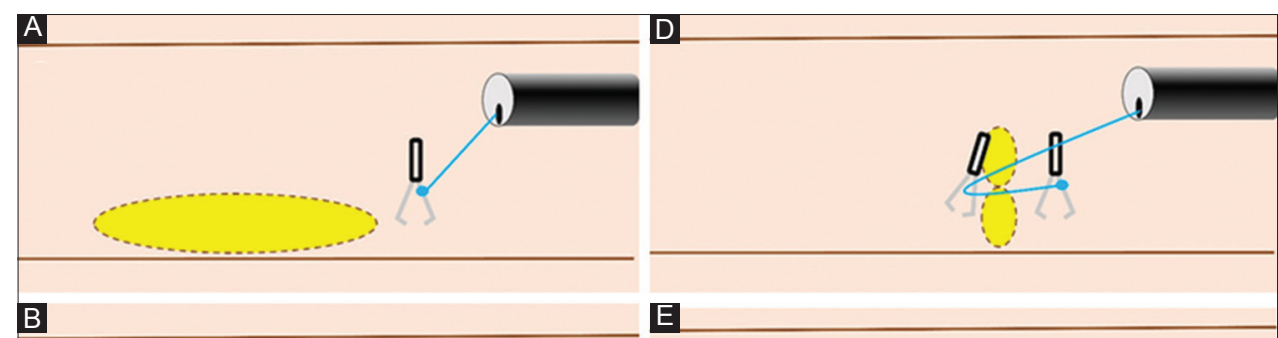

B

E
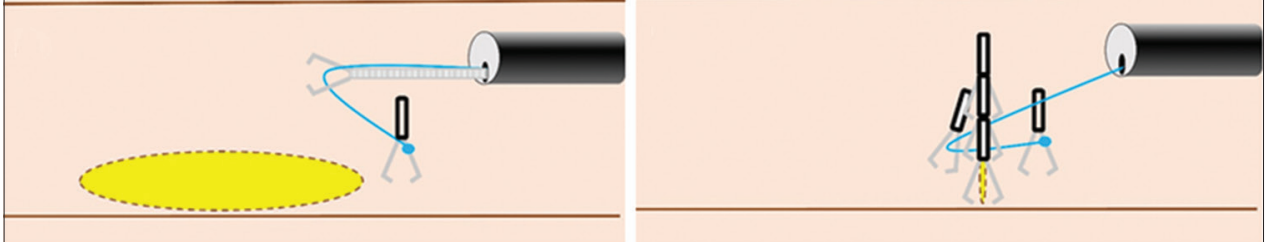

C

$\mathbf{F}$

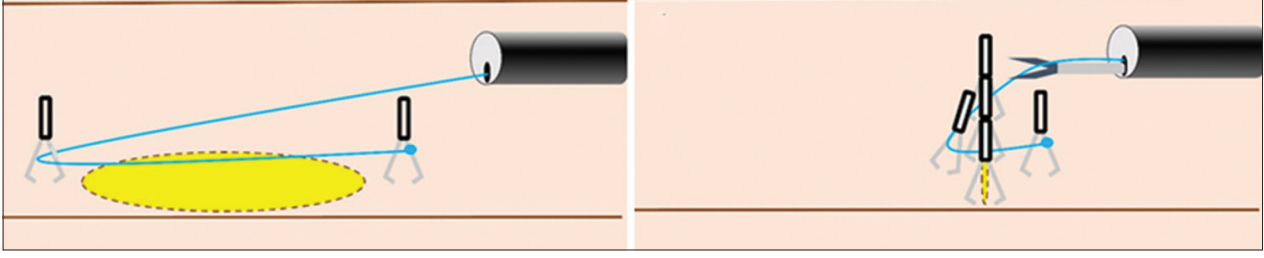

Figure 4 Line-assisted complete closure method. (A) A clip with a nylon fishing line is applied through the working channel of a videoendoscope to the mucosa on the proximal side of the defect. (B, C) A second clip without line is applied to the mucosa on the distal side of the defect, anchoring the line of the first clip. (D) The clips are then gathered by pulling on the line. (E) Additional clips are applied to make the closure secure and tight. (F) After complete closure, the line is cut with scissor forceps

performed the procedures in an operating room with the patient under general anesthesia, and this approach was mandatory for the EFTR procedure. In this environment we were able to perform a stable procedure and, in cases where defect closure was impossible or there were other complications, could ask for prompt intervention from other surgeons.

In Japan, almost all medical services must adhere to the national health insurance system, whose coverage for resection of gastric GISTs is limited to surgery and LECS. National health insurance coverage for EFTR of GISTs will promote the wider dissemination of this treatment.

This study had a few limitations. First, because of its retrospective nature, selection bias must exist. However, we regarded this study as a preliminary exploratory investigation. After establishment of practice according to this observation, the method will be validated in a prospective study. Second, this study was a single-arm descriptive study. Although comparison between those treated by LECS or laparoscopic surgery must be recognized as important information, we considered that this small study sample was not suitable for adjusted comparisons such as multivariate analysis or propensity score matching analysis. In this regard, a large-scale multicenter comparative study is warranted in the future. Third, we evaluated only short-term outcomes, despite the presence of cases (5 of 8) with a histologically indeterminate resection margin (RX). A favorable outcome has been reported in a Chinese longterm follow-up study [14]. Long-term follow-up studies in a Japanese cohort are required to justify this treatment approach.

In conclusion, EFTR of small $(\leq 4 \mathrm{~cm})$ intraluminal growth type GISTs shows every sign of being practical in Japanese

\section{Summary Box}

\section{What is already known:}

- Gastrointestinal stromal tumors (GISTs) are potentially malignant and are indicated for resection

- Currently the standard resection method for gastric GISTs is surgery with negative surgical margins

- Although many studies of endoscopic treatment for submucosal tumors in other countries have been published, little has been reported from Japan

\section{What the new findings are:}

- In this retrospective observational study, we investigated small $(\leq 4 \mathrm{~cm})$ intraluminal growth type GISTs treated by only peroral flexible endoscopy

- Endoscopic resection for selected cases of small intraluminal GISTs is feasible

- Endoscopic resection would be a viable alternative treatment option to laparoscopic surgery

practice, warranting further investigation of its feasibility in a large-scale prospective study. 


\section{Acknowledgments}

We express our sincere gratitude to Prof. Ping-Hong Zhou and Dr. Jian-Wei Hu for their generous teaching of the skill of EFTR. We also thank Hugh McGonigle, from Edanz Group (www.edanzediting.com/ac), for editing a draft of the manuscript. Finally, we thank Chihiro Tsunoda and Minoru Kato for illustrating Fig. 3 and 4, respectively.

\section{References}

1. Japanese Clinical Practice Guidelines for Gastrointestinal Stromal Tumors (GIST), Third Edition. Kanehara \& Co., Ltd. Tokyo, 2014.

2. Demetri GD, Benjamin RS, Blanke CD, et al; NCCN Task Force. NCCN Task Force report: management of patients with gastrointestinal stromal tumor (GIST) - update of the NCCN clinical practice guidelines. J Natl Compr Canc Netw 2007;5(Suppl 2):S1-S29.

3. Casali PG, Abecassis N, Aro HT, et al; ESMO Guidelines Committee and EURACAN. Gastrointestinal stromal tumours: ESMO-EURACAN Clinical Practice Guidelines for diagnosis, treatment and follow-up. Ann Oncol 2018;29:iv68-iv78.

4. Nishimura J, Nakajima K, Omori T, et al. Surgical strategy for gastric gastrointestinal stromal tumors: laparoscopic vs. open resection. Surg Endosc 2007;21:875-878.

5. Otani Y, Furukawa T, Yoshida M, et al. Operative indications for relatively small $(2-5 \mathrm{~cm})$ gastrointestinal stromal tumor of the stomach based on analysis of 60 operated cases. Surgery 2006;139:484-492.

6. Novitsky YW, Kercher KW, Sing RF, Heniford BT. Long-term outcomes of laparoscopic resection of gastric gastrointestinal stromal tumors. Ann Surg 2006;243:738-745; discussion 745-747.

7. Privette A, McCahill L, Borrazzo E, Single RM, Zubarik R. Laparoscopic approaches to resection of suspected gastric gastrointestinal stromal tumors based on tumor location. Surg Endosc 2008;22:487-494.

8. Chen T, Zhou PH, Chu Y, et al. Long-term outcomes of submucosal tunneling endoscopic resection for upper gastrointestinal submucosal tumors. Ann Surg 2017;265:363-369.

9. Lv XH, Wang CH, Xie Y. Efficacy and safety of submucosal tunneling endoscopic resection for upper gastrointestinal submucosal tumors: a systematic review and meta-analysis. Surg Endosc 2017;31:49-63.

10. Li QL, Chen WF, Zhang C, et al. Clinical impact of submucosal tunneling endoscopic resection for the treatment of gastric submucosal tumors originating from the muscularis propria layer (with video). Surg Endosc 2015;29:3640-3646.

11. Zhang C, Hu JW, Chen T, et al. Submucosal tunneling endoscopic resection for upper gastrointestinal multiple submucosal tumors originating from the muscular propria layer: a feasibility study. Indian J Cancer 2015;51(Suppl 2):e52-e55.

12. Hu JW, Zhang C, Chen T, et al. Submucosal tunneling endoscopic resection for the treatment of rectal submucosal tumors originating from the muscular propria layer. J Cancer Res Ther 2014;10(Suppl):281-286.

13. Xu MD, Cai MY, Zhou PH, et al. Submucosal tunneling endoscopic resection: a new technique for treating upper GI submucosal tumors originating from the muscularis propria layer (with videos). Gastrointest Endosc 2012;75:195-199.

14. Yu C, Liao G, Fan C, et al. Long-term outcomes of endoscopic resection of gastric GISTs. Surg Endosc 2017;31:4799-4804.

15. Huang LY, Cui J, Lin SJ, Zhang B, Wu CR. Endoscopic full-thickness resection for gastric submucosal tumors arising from the muscularis propria layer. World J Gastroenterol 2014;20:13981-13986.
16. Zhou PH, Yao LQ, Qin XY, et al. Endoscopic full-thickness resection without laparoscopic assistance for gastric submucosal tumors originated from the muscularis propria. Surg Endosc 2011;25:2926-2931.

17. Inoue $\mathrm{H}$, Ikeda $\mathrm{H}$, Hosoya $\mathrm{T}$, et al. Submucosal endoscopic tumor resection for subepithelial tumors in the esophagus and cardia. Endoscopy 2012;44:225-230.

18. Liao GQ, Chen T, Qi XL, et al. Laparoscopic management of gastric gastrointestinal stromal tumors: A retrospective 10-year singlecenter experience. World J Gastroenterol 2017;23:3522-3529.

19. Takeuchi Y, Uedo N, Ishihara R, et al. Efficacy of an endo-knife with a water-jet function (Flushknife) for endoscopic submucosal dissection of superficial colorectal neoplasms. Am J Gastroenterol 2010;105:314-322.

20. Kobara H, Mori H, Nishiyama N, et al. Over-the-scope clip system: a review of 1517 cases over 9 years. J Gastroenterol Hepatol 2019;34:22-30.

21. Tashima T, Ohata K, Sakai E, et al. Efficacy of an over-the-scope clip for preventing adverse events after duodenal endoscopic submucosal dissection: a prospective interventional study. Endoscopy 2018;50:487-496.

22. Qiao Z, Ling X, Zhu J, et al. Therapeutic application of purse-string sutures with nylon loops and metal clips under single-channel endoscopy for repair of gastrointestinal wall defects. Exp Ther Med 2018;15:4356-4360.

23. Shi Q, Chen T, Zhong YS, et al. Complete closure of large gastric defects after endoscopic full-thickness resection, using endoloop and metallic clip interrupted suture. Endoscopy 2013;45:329-334.

24. Kato M, Takeuchi Y, Yamasaki Y, et al. Technical feasibility of lineassisted complete closure technique for large mucosal defects after colorectal endoscopic submucosal dissection. Endosc Int Open 2017;5:E11-E16.

25. Shichijo S, Yamasaki Y, Takeuchi Y. Case of colonic adenoma involving a diverticulum resected by a traction-assisted endoscopic submucosal dissection technique. Dig Endosc 2017;29:729-730.

26. Yamasaki Y, Takeuchi Y, Kato M, Uedo N, Ishihara R. Line-assisted complete closure of large gastric mucosal defects by use of multiple clip-and-line technique. VideoGIE 2016;1:49-50.

27. Japanese Gastric Cancer Association. Japanese classification of gastric carcinoma: $3^{\text {rd }}$ English edition. Gastric Cancer 2011;14:101-112.

28. National Cancer Institute NIoH, U.S. Department of Health and Human Services. Common Terminology Criteria for Adverse Events (CTCAE) v5.0. 2017.

29. DeMatteo RP, Lewis JJ, Leung D, Mudan SS, WoodruffJM, Brennan MF. Two hundred gastrointestinal stromal tumors: recurrence patterns and prognostic factors for survival. Ann Surg 2000;231:51-58.

30. Fong Y, Coit DG, Woodruff JM, Brennan MF. Lymph node metastasis from soft tissue sarcoma in adults. Analysis of data from a prospective database of 1772 sarcoma patients. Ann Surg 1993;217:72-77.

31. McCarter MD, Antonescu CR, Ballman KV, et al; American College of Surgeons Oncology Group (ACOSOG) Intergroup Adjuvant Gist Study Team. Microscopically positive margins for primary gastrointestinal stromal tumors: analysis of risk factors and tumor recurrence. J Am Coll Surg 2012;215:53-59; discussion 59-60.

32. Suzuki S, Gotoda T, Kobayashi Y, et al. Usefulness of a traction method using dental floss and a hemoclip for gastric endoscopic submucosal dissection: a propensity score matching analysis (with videos). Gastrointest Endosc 2016;83:337-346.

33. Halvax $\mathrm{P}$, Diana $\mathrm{M}$, Lègner $\mathrm{A}$, et al. Endoluminal full-thickness suture repair of gastrotomy: a survival study. Surg Endosc 2015;29:3404-3408.

34. Mori H, Kobara H, Fujihara S, et al. Innovative pure non-exposed endoscopic full-thickness resection using an endoscopic suturing device. Gastrointest Endosc 2016;84:178-179.

35. Yamamoto $Y$, Uedo N, Abe N, et al. Current status and feasibility of endoscopic full-thickness resection in Japan: Results of a questionnaire survey. Dig Endosc 2018;30(Suppl 1):2-6. 